\title{
THE EARLY MANIFESTATIONS AND COURSE OF DIPLEGIA IN CHILDHOOD
}

\author{
BY \\ T. T. S. INGRAM \\ From the Department of Child Life and Health, University of Edinburgh
}

(RECEIVED FOR PUBLICATION OCTOBER 21, 1954)

During a recent survey of children suffering from cerebral palsy in Edinburgh it was found that diplegia was relatively infrequently diagnosed before the age of 2 years. The average delay between the doctor seeing the child for the first time, on account of manifestations of diplegia, and the diagnosis being reached was over one year. The classical picture of diplegia presented in the textbooks is of a child affected by rigidity, spasticity and contractures of the limbs, and only when these features were present was the diagnosis made in the majority of patients. Yet the symptoms and signs of the earlier stages of diplegia are typical and should enable the diagnosis to be made in most patients long before contractures have occurred. In the present paper the early manifestations and course of diplegia will be discussed.

The terminology and classification used during the survey has been described in an earlier article (Ingram, 1955). The term diplegia is used to describe a condition of more or less symmetrical paresis of cerebral origin, more severe in the lower limbs than in the upper and dating from birth or shortly after. Mental deficiency, epilepsy and strabismus are frequently associated findings. Patients suffering from diplegia associated with cerebellar ataxia are not considered in this article.

\section{The Case Material}

The findings described in the present article are those of 79 patients with diplegia who were ascertained during a survey of children suffering from cerebral palsy in Edinburgh during 1953. They varied in age from a few weeks to 15 years and included 49 males and 30 females, figures which give a similar sex distribution to that found by Asher and Schonell (1950). The cases were ascertained from a very large number of sources and the incidence found was 0.75 cases of diplegia per 1,000 of the population under the age of 15 years. This figure is somewhat higher than similar incidence figures obtained by other regional surveys. It seems probable that the patients may be regarded as being representative of children suffering from diplegia in the community.

The diplegia was thought to be of congenital origin in all but one of the 79 patients, and the first manifestations had been noted in 64 by parents or guardians before the children were 18 months old. Fifty-eight of these patients were taken to their doctors before this age, yet the diagnosis was reached in only 18 by the age of 18 months and in 45 by the age of 2 years. In 14 patients the diagnosis was reached only when the child was over 4 years old (Table 1).

TABLE 1

AGES AT TIME OF FIRST SIGN OF ABNORMALITY, THE CORRECT DIAGNOSIS, FIRST VISIT TO DOCTOR'AND FIRST CLINIC REFERRAL IN 79 PATIENTS WITH DIPLEGIA

\begin{tabular}{|c|c|c|c|c|}
\hline Age & $\begin{array}{c}\text { First } \\
\text { Aboormality } \\
\text { Noted by } \\
\text { Parents }\end{array}$ & $\begin{array}{l}\text { First } \\
\text { Visit to } \\
\text { Doctor }\end{array}$ & $\begin{array}{c}\text { First } \\
\text { Clinic } \\
\text { Referral }\end{array}$ & $\begin{array}{l}\text { Correct } \\
\text { Diagnosis }\end{array}$ \\
\hline $\begin{array}{l}\text { Under } 6 \text { months } \\
\text { Over } 6 \text { months. }\end{array}$ & 31 & 23 & 8 & 3 \\
\hline $\begin{array}{l}\text { under } 1 \text { year... } \\
\text { Over } 1 \text { year, under }\end{array}$ & 23 & 10 & 12 & 1 \\
\hline & 10 & 25 & 12 & 14 \\
\hline $\begin{array}{l}\text { under } 2 \text { years } \\
\text { Over } 2 \text { years, }\end{array}$ & 11 & 12 & 12 & 27 \\
\hline $\begin{array}{l}\text { under } 4 \text { years } \\
\text { Over } 4 \text { years } \\
\text { Unknown }\end{array}$ & $\begin{array}{l}2 \\
0 \\
2\end{array}$ & $\begin{array}{l}7 \\
0 \\
2\end{array}$ & $\begin{array}{r}26 \\
8 \\
1\end{array}$ & $\begin{array}{r}19 \\
14 \\
1\end{array}$ \\
\hline Totals & 79 & 79 & 79 & 79 \\
\hline
\end{tabular}

The Stages of Diplegia

The majority of patients with diplegia do not show the classical picture of muscular rigidity or spasticity until some time after birth. In fact, after the immediate effects of marked prematurity or birth trauma have worn off the babies are frequently regarded as being normal, even when carefully followed up by infant clinics. That there should be a delay, sometimes of months, in the appearance of dramatic symptoms and signs has led some authors 
to state that diplegia is frequently a condition of post-natal aetiology.

Unfortunately few of the classical authors studied the early stages of diplegia closely, though a few showed some interest in its early manifestations (Little, 1862; Parrot, 1873; Lovett, 1888; Osler, 1889). More recently, however, increasing attention has been focused on the nature and significance of postural reflexes which are transiently present in normal infants but which tend to persist, sometimes for years, in children suffering from diplegia. It has been increasingly realized that the neurological findings, and especially the postural reflexes, change as the patient grows older and as his nervous system matures (Bobath and Bobath, 1950).

The change from the infant of a few weeks old who shows little demonstrable abnormality on neurological examination to the child 2 or 3 years old with flexion contractures of all four limbs is a gradual one. But the sequence of changes that occurs in the neurological findings marking the child's progress to this crippled state is remarkably constant. For the purposes of description three stages may be recognized in the gradual development of this final picture of spasticity with contracture of the limbs: the hypotonic stage, the dystonic stage and a third stage in which rigidity and spasticity are present together in varying degree.

The Hypotonic Stage. In the first few weeks after birth the majority of children with diplegia show little obvious abnormality related to the neurological disorder. From the mothers or guardians of the 79 patients in the present series, however, a history was obtained in 31 which suggested that the infants were hypotonic in the first weeks after delivery. 'His head flopped all over the place.' 'He felt like a half-filled pillow for weeks and weeks', were typical comments. At the same time a high proportion of patients were noted to show a marked poverty of movement. The child would retain any position in which he was laid down for many hours. 'He was so still, you wouldn't think he had breathed all night,' said one mother.

Five diplegic patients have been examined in the stage of hypotonia; one of these is included in the series and four are excluded, because they lived outside Edinburgh or were born after the survey was completed. Poverty of movement was observed, especially in the trunk and proximal parts of the limbs. Apart from small-range, feeble finger movements, the patients were very still. On passive movements of the limbs and when the children were held in the arms generalized muscular hypotonia was evident. In all five children the Moro responses and grasp reflexes were more clearly defined and easily elicited than in normal children. The tonic neck reflexes were not exaggerated, and appeared to be less marked than normal in two of the patients. The duration of the stage of hypotonia is very variable and patients were encountered in this stage whose ages varied from 6 weeks to 15 months. In 28 of the 31 patients in whom a history of a hypotonic stage was elicited it lasted between six weeks and six months.

The Dystonic Stage. At about the time the diplegic child first attempts to hold up the head a change occurs in this picture of little apparent abnormality. The mother finds that when she changes the child's position abruptly, especially when dressing or washing him, he suddenly becomes stiff in her arms. The neck and back are extended. The upper limbs are adducted and internally rotated at the shoulder, extended at the elbows, pronated at the forearms and semiflexed at the wrist and fingers. The lower limbs are extended, adducted and somewhat internally rotated at the hips, extended at the knees, plantar flexed at the ankles and flexed at the toes (Fig. 1). This position, essentially one of opisthotonos, is achieved by a sudden dystonic movement of the trunk and neck associated with extensor movements of the limbs.

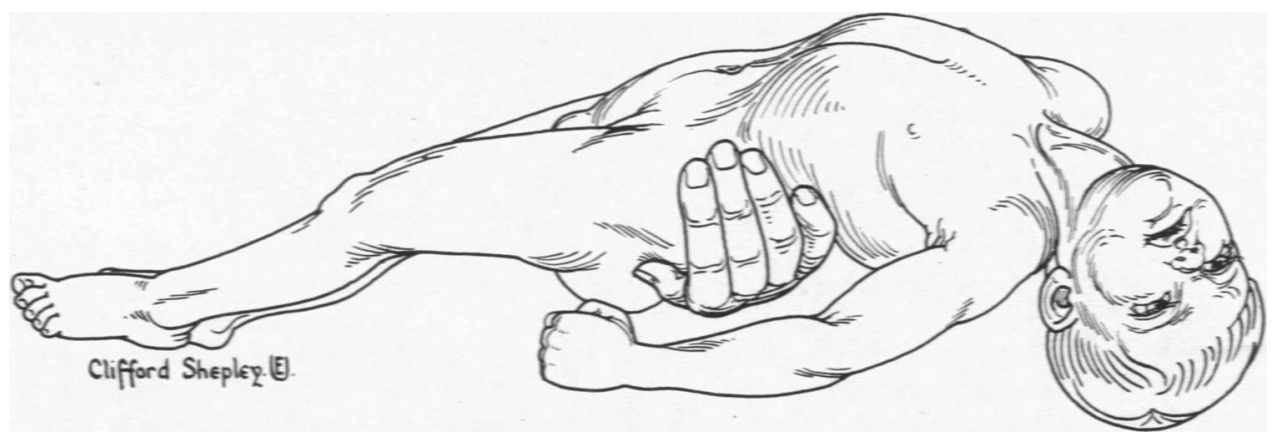

FKG. 1.-The position assumed during dystonic attacks by a severely affected diplegic child aged 15 months. 
The position is maintained for only a few seconds, during which time the tone of the muscles is very greatly increased and the whole child is rigid. Sometimes there appears to be a slight impairment of consciousness during the attacks but it is never lost. Initially the attacks occur only once or twice a day, but over the course of one or two weeks their frequency increases. They become more easily produced and may occur as often as $\mathbf{2 0}$ or $\mathbf{3 0}$ times a day, greatly distressing the child. Frequently dystonic attacks are diagnosed as being epileptiform but they respond to none of the commonly used anticonvulsive drugs.

On examination at the height of the dystonic stage, it is usually found that the child is beginning to hold up the head, but is still unable to hold it steadily. He makes little attempt to reach for objects and poverty of voluntary movement is still evident. The tone of the limbs remains hypotonic at rest. The grasp reflexes are brisk. With care not to extend the head suddenly the Moro responses may be demonstrated in most of the patients. When the child is held vertically there is rigid increase of tone in the limbs. This is more marked in the lower than in the upper limbs. The lower limbs show a position of extension at the knees and plantar flexion at the ankles. The hips are adducted, slightly internally rotated and may show a position of full extension or of a few degrees of flexion. The legs may scissor. When the child's head is briskly extended, or he is suddenly rotated into the horizontal position from the vertical, supported by a hand in the small of the back, there is a sudden gross increase of tone in the trunk and limbs, associated with a quick, writhing, dystonic movement of the trunk. The limbs extend and a position of opisthotonos is assumed for a few seconds. This position corresponds exactly with that described by the mothers as resulting from changes in the position of the child when she handles him.

After a variable period the dystonic attacks become less easily produced and less frequent. In the majority of patients the generalized dystonic attacks are present for between two and six months, but in others the period may exceed one year. As the attacks begin to be less frequent the mother notices for the first time that the limbs are constantly rigid. This is first evident in the thigh or calf muscles and she may find that she has to use force to separate the thighs when placing the napkin, or even pass it between them on the end of a pencil. When she washes the lower limbs they feel stiff and unyielding. In the course of a few weeks the rigidity becomes increasingly evident and spreads to involve the upper limbs in triplegic and tetraplegic cases.
On examination during the period when dystonic attacks are waning a constant muscular rigidity is found, which is more severe in the lower limbs than in the upper. In the majority of patients sudden extension of the head still gives rise to a typical dystonic attack. In a few patients, however, it is necessary to extend the head and put pressure on the soles of the feet before dystonia is produced. When the child is made to exert himself, or is distressed, the affected limbs tend to assume extensor positions even if true dystonic movements of the trunk have ceased to occur.

During the survey six patients were classified as being in the dystonic stage of diplegia. Another seven with dystonia have been seen who are not included in the survey. In all, the essentially opisthotonic position described above could be produced by suddenly extending the head and putting pressure on the soles of the feet. Of the 79 children with diplegia in the survey, 39 had evidence of having had dystonic attacks, either in the history or on examination. It was noted that a history of dystonia was found much less frequently in older children than in younger and it is probable that if mothers' memories had been longer a greater number of patients who had had dystonic attacks would have been ascertained.

The Phase of Rigidity. The third stage of diplegia is conveniently divided into two phases for the purposes of description, first the phase of rigidity and secondly the phase of spasticity.

As has been described, the phase of rigidity emerges gradually from the stage of dystonia and for a period, as rigidity increases in extent and severity, dystonic attacks persist. Somewhat arbitrarily the phase of rigidity may be said to begin, for purposes of classification, when generalized dystonic attacks involving the neck, trunk and limbs cease to occur as a result of changes in the child's position. This is usually about the time he attempts to sit with support with the knees flexed and is beginning to use the upper limbs in order to reach for objects. The parents notice that the attempts to grasp are clumsy and that the child appears to hold toys at arm's length with the affected upper limbs extended. In the majority of the patients in the series generalized dystonic attacks ceased between the ages of 5 and 12 months.

On examination children with predominant rigidity show clumsiness of voluntary movements. Manipulations with the hands are immature in type, all the fingers being used together as a unit opposed to the palms. The limbs tend to be held in positions of mild extension at rest, but this tendency is greatly 
increased by exertion, the performance of voluntary movements, extension of the head or pressure on the soles of the feet, especially when the child is held erect. When an affected upper limb is moved voluntarily it tends to adduct at the shoulder, extend at the elbow, pronate at the forearm and flex at the wrist and fingers. This is the reason for the impression that the child is holding objects

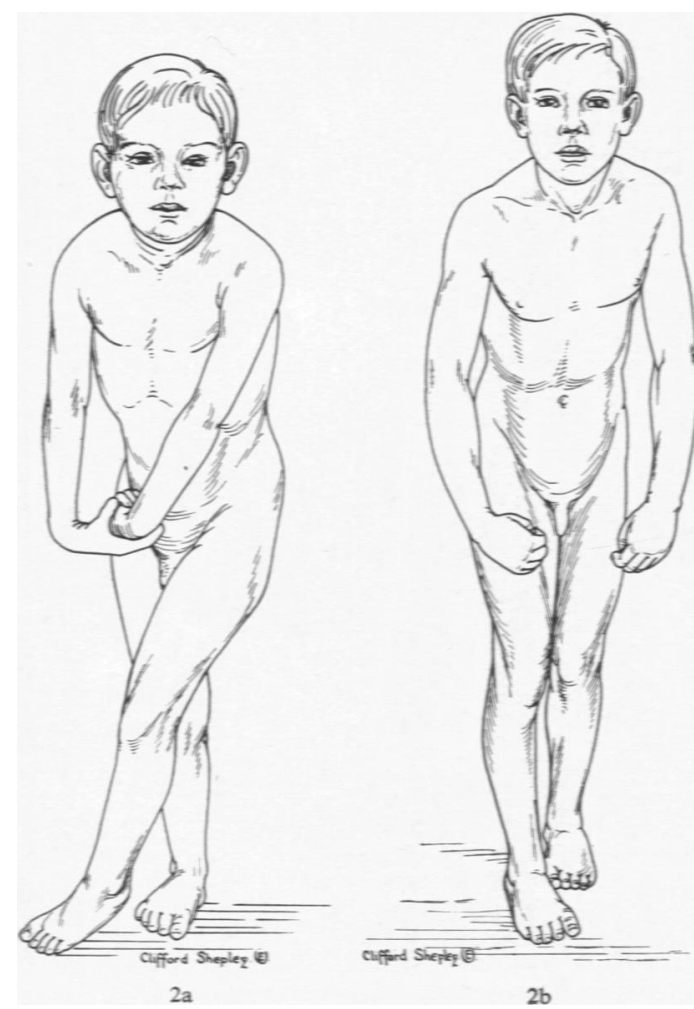

Figs. $2 \mathrm{a}$ and $2 \mathrm{~b}$.- Tetraplegic children, aged 4 and 6 years respectively, showing the extended positions assumed by the limbs in the phase of rigidity.

at arm's length. If the contralateral limb is also involved it too will show a tendency to attain a similar position to the operating limb and the lower limbs also extend. Thus associated movements of extension are present in all the other limbs when one is used and in all the limbs involved when the child exerts himself by straining or attempting to change his position. These associated movements are similar to those observed in the limbs during the earlier dystonic attacks. In the latter, however, the neck and trunk also took part and the involuntary movement was more rapid, more extensive and more powerful. In the stage of rigidity the involuntary postural activity appears to become more inhibited. Nevertheless it is found that the manoeuvres which were most potent in causing dystonic attacks at the earlier stage are also those which produce associated movements of limb extension most readily. When the child is held in the vertical position great rigidity of the lower limbs is evident and they are held scissored and either extended at the hips or in a few degrees of flexion. The knees are extended, the feet plantar flexed. The rigid increase of tone in the upper limbs is less accentuated and their positions may not be markedly constrained. If the feet are placed on the floor, however, or pressure is applied to the soles of the feet, the involved upper limbs immediately assume a position similar to that found during the earlier dystonic attacks.

Involuntarily the child tends to hold the head well forward with the neck flexed. The maintenance of this position minimizes the danger of sudden head extension occurring, with its consequent reflex extension of the limbs and disturbance of posture (Fig. 2). Walking tends to be impossible for a long time, because this involves pressure on the soles of the feet and whenever a step is taken some extension of the neck is almost inevitable. As a result there is involuntary extension of the lower limbs and sometimes the back, which makes the child lose his balance and is the origin of the extensor thrust, noted by physiotherapists, which may last even into the phase of predominant spasticity. The children usually learn to sit without support with the knees flexed, though not with them extended during the phase of rigidity. Any excessive exertion, however, is liable to result in extension of the lower limbs so that if the child is on a chair he is dragged forwards by pressure of the back of the thighs on the chair edge and pulled to the horizontal position.

During the predominantly rigid phase of diplegia the tendon jerks are usually sluggish and difficult to elicit. The Hoffmann responses are not obtained, and the true Babinski response is hardly ever found. The tonic neck reflexes are positive, but extension of the limbs is much more readily produced than flexion. The grasp reflexes are usually present. The Moro response may be sluggishly present in a few patients but it is never brisk and in the majority it disappears completely during the stage of rigidity.

The Spastic Phase. This phase is apparent only after rigidity of the muscles has been present for some weeks or months. In some spasticity appears only after the child has shown predominant rigidity for many years. Its onset is insidious and is usually apparent about the time the child is beginning to 
use the thumb and fingers a little and has learnt to sit without support with the knees flexed. Usually attempts to pull himself into the erect position follow shortly after this time.

The first evidence that spasticity has made its appearance is commonly a change in the nature of the associated movements of the limbs which results from voluntary activity or exertion. Over a period of a few weeks the parents notice that instead of being straight the upper limbs tend to be flexed and that all movements are less clumsy and jerky, though they may be slower than before. A grandmother noted, 'His arms used to look as if they'd been put on the wrong way round but then they became all bent up'. The same tendency to flexor positions rather than extensor positions becomes apparent in the lower limbs as in the upper. Unless the child is encouraged in all activities at the stage of early spasticity, especially standing and walking with support, flexion contractures rendering movement impossible can ensue in a few weeks. One nurse in an institution for the mentally defective summarized the position when talking of a bedridden tetraplegic patient, 'First he was too daft to move and now he can ${ }^{\prime \prime}$.

On examination the findings in patients showing spastic increase of tone in the limbs depend very much on the relative severity of rigidity and superimposed spasticity in the individual patient. In patients who remain rigid, associated movements will remain extensor in type, and in patients who show early spasticity flexor associated movements make an early appearance. In the majority of diplegic patients, however, there is a transitional period during which spasticity is increasing in the limbs and the associated movements are neither typically extensor nor typically flexor.

By the time spasticity is well marked in the limbs, not only are the associated movements predominantly flexor but there is also a tendency for the child to hold the limbs in flexed positions at rest. These positions become exaggerated when the child exerts himself. Thus when he walks or attempts to walk there is flexion of the fingers, wrist and elbows, adduction of the thumbs, pronation of the forearms, and adduction and internal rotation of the shoulders in the affected upper limbs. An increase of flexion at the hips and knees, adduction of the thighs and plantar flexion also become evident. The typical walking position of the tetraplegic child results. The legs are scissored, the knees are semiflexed and the heels are not placed to the ground. The child appears to be leaning forwards with the upper limbs flexed across the chest (Fig. 3). When a spastic upper limb is used for grasping athetosis is sometimes evident in the fingers and wrist, though this is not usually marked. Both in the operating and contralateral upper limb the flexor associated movements tend to be most marked at the distal joints in contrast to the extensor associated movements of the rigid phase, which are most marked in the proximal parts of the limbs.

The tonic neck reflex is very marked in the

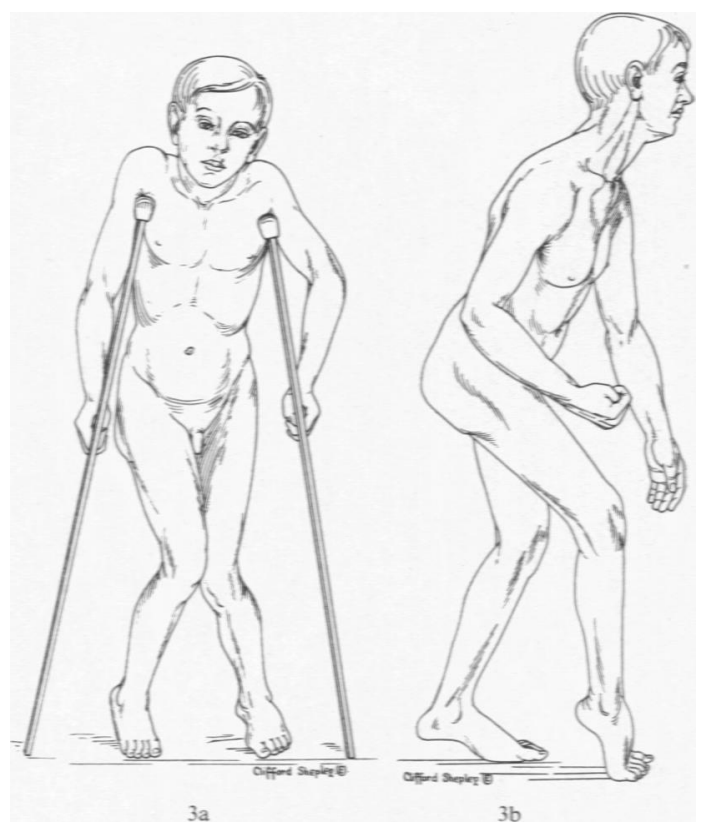

FKGS. 3a and 3b.-Tetraplegic children, aged 10 and 15 years respectively, showing the flexor associated movements and contractures of the limbs in the phase of predominant spasticity.

majority of patients in the spastic phase and both flexor and extensor positions of the upper limbs are readily demonstrable in triplegic and tetraplegic cases. The Moro response is absent and the grasp reflex present only occasionally. The tone of the limbs, and especially of the lower limbs, is a mixture of rigidity and spasticity, which gives rise to a very typical feel when they are moved passively. Stretch responses may be demonstrated in the muscles of the affected limbs, first and most easily in the adductors of the thighs and in the calf muscles. The tendon jerks are exaggerated, always to a greater extent in the lower limbs than the upper, and ankle and patellar clonus is frequent. The Hoffmann responses are frequently positive. The plantar responses are extensor bilaterally.

Once spasticity is evident, contractures in flexion are very liable to occur in the affected limbs (Fig. 3). Contractures give rise to an increase of rigidity 
which is accentuated as the limbs are moved passively from the flexed to the extended position, while the earlier rigidity of the limbs tended to be constant throughout the range of passive movement. The contracture is most likely to be severe in the lower limbs in diplegic patients, and it results, when severe, in a great diminution of the stretch reflexes and the tendon jerks. In those all too numerous patients in whom repeated tendon lengthenings, muscle transplants and joint fixations have been performed, the true neurological findings may be further concealed.

\section{The Relationship of the Stages of Diplegia to Each Other}

The importance of recognizing that the findings in diplegia change in a regular manner as the condition develops is that this leads not only to earlier diagnosis, but also to better understanding of some of the variations which are encountered in the final clinical picture. The stages of diplegia mark definite milestones in the development of the child's motor control. Thus a child who shows dystonic attacks at the age of 4 years and is, therefore, in the stage of dystonia, must be regarded as showing a very immature motor control. On the other hand, a child in the phase of spasticity has passed through all the stages of diplegia and his neuromuscular mechanisms may be regarded as being more mature, though still disordered. Unless severely affected by contracture, he will be capable of much more in the way of controlled voluntary movement of the limbs than any patient in the stage of dystonia.

The findings in the different limbs of patients with diplegia are not always those of the same stage. Thus it is quite common, even in children aged 10 or 12 , to find that one limb is predominantly rigid while the other limbs are predominantly spastic. The child who holds one arm behind his back in the extended position in order to limit the range of its involuntary postural movements while walking and holds the other like a hemiplegic arm across his chest is not uncommon. Almost invariably the spastic limb is the more useful, unless contracture is severe.

It must be stressed that the stages of diplegia are determined by the relative maturity of the child's nervous system much more than by age. Thus one child was seen at the stage of hypotonia at the age of 2 years and one showed spastic diplegia at the age of 10 months. Nevertheless it is of some value to indicate the ages at which the majority of patients show the different stages. Of the 79 children in the present series, a history suggestive of hypotonia was elicited in 31 . In 20 of these this stage lasted less than six months, and in 11 less than four months from the time of birth. The dystonic stage was noted in 39 patients. In 29 of these, it was evident before the age of 6 months and in eight between the ages of 6 months and 1 year. Only two patients gave a history which suggested that the dystonia had begun over the age of 1 year. The duration of the dystonic stage was unknown in four patients who were still showing dystonic attacks when the survey closed. In the remaining 35 patients it lasted less than six months in 19 patients and between six months and one year in 11. In five patients the duration of the stage of dystonia was over one year. The age at which rigidity was first evident varied from 10 weeks to three years. In about half of the 72 rigid or spastic patients in the survey, no history of a previous dystonic stage was elicited, though that is not to say that it had not been present.

As has been noted, a few patients showed persistent rigidity throughout their lives without spasticity being superimposed upon this stage. A small number of patients appeared to develop spasticity at a relatively early age without a history of either previous dystonia or rigidity. The majority of patients, however, showed the sequence of stageshypotonic, dystonic, rigid and spastic-which has been described. It was, therefore, much more usual to encounter cases showing mixed rigid and spastic increase of tone in the limbs than cases showing only rigidity or spasticity.

The age at which spasticity was first evident wass even more variable than the age at which dystonia or rigidity first appeared. It was unusual to find spasticity before the age of 8 months, and in the majority of patients it made its first appearance between the ages of 10 and 18 months. On the other hand, the speed with which contracture could cripple the child with spastic diplegia was remarkable. One child was unable to extend either elbow beyond the right angle or pronate either forearm by the age of 18 months, though spasticity had made its appearance only at the age of 11 months so far as could be ascertained from the history.

\section{The Course of Diplegia Related to the Extent and Severity of the Paresis}

The severity of diplegia in any case may be assessed roughly by considering the stage of the condition reached at a given age and the extent of the limb involvement. As might be expected, the stages of diplegia are most manifest in patients with severe tetraplegic involvement; they are much less clearly defined in patients in whom the upper limbs 
are spared. In particular it is unusual to obtain a history of severe generalized dystonic attacks in paraplegic patients. It seems probable that this is due to the fact that they are much slighter and the duration of the dystonic stage is shorter than in patients with triplegic or tetraplegic involvement. In two paraplegic patients, not included in the survey, dystonic attacks were easily elicited by sudden head extension and pressure on the soles of the feet though in neither was a history obtained which suggested that the children had shown a dystonic stage. In one case dystonia could be produced for only three weeks and in the other for only five. Clearly with slight attacks occurring for such a short period retrospective history taking must frequently fail.

In the present series of 79 patients with diplegia, there was evidence of a dystonic stage either in the history or on physical examination in 19 of the 27 patients with tetraplegia, in 15 of the 23 patients with triplegia and of the 29 patients with paraplegia. At the time of the survey one patient was in the stage of hypotonia, six in the stage of dystonia and 72 were in the stage of rigidity or spasticity. Since the majority of the patients were seen on a number of occasions it was possible to observe the progression of some from stage to stage.

The Early Manifestations of Diplegia

In Table 2 are indicated the commonest initial

TABLE 2

NATURE OF FIRST NEUROLOGICAL MANIFESTATIONS RESULTING IN MEDICAL HELP IN 79 CHILDREN WITH DIPLEGIA

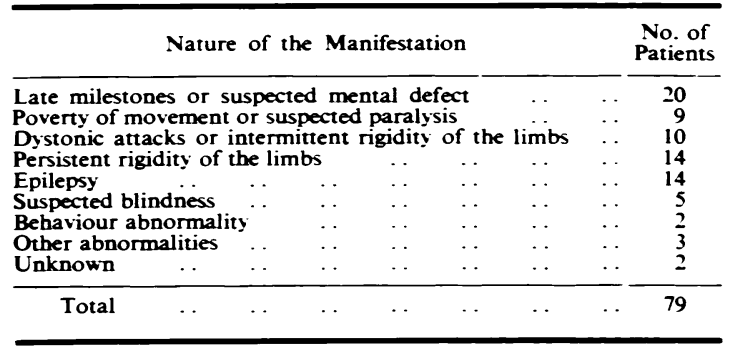

manifestations of diplegia noted by the parents in 79 patients in the present series. It is apparent that in most cases these should have resulted in a full neurological examination of the patient. If the patient had been fully examined neurologically and the effects of sudden extension of the head been investigated with the child in the erect and supine positions, the diagnosis of diplegia would have been made more frequently at an early age. If diplegia could be diagnosed earlier, before the stage of rigidity or spasticity, the danger of permanent disablement being caused by contractures would be lessened.

\section{Summary}

During a recent survey of children suffering from cerebral palsy it was found that the average delay in a diagnosis of diplegia being made following the first visit to a doctor was over one year. This delay appeared to be due to ignorance of the early manifestations to be expected in diplegia befere the late stage of rigidity or spasticity with contractures.

The early manifestations of diplegia are described as they were encountered during the survey. It is suggested that the majority of patients pass through a stage during which they show hypotonia and a stage during which they show sudden generalized dystonic attacks on changes of position before rigidity or spasticity is evident. The stage of dystonia seems to be of shorter duration and the dystonic attacks less marked in patients with paraplegic involvement than in those with triplegia or tetraplegia. It is suggested that the diagnosis would be reached earlier in patients with diplegia if a neurological examination, including attempts to produce dystonia, was made in every suspected case.

I am grateful to the many physicians and surgeons in Edinburgh who allowed me ready access to their patients and case notes. I must thank the staff of the Scottish Council for the Welfare of Spastics for their interest and encouragement, especially Dr. Charles Balf, Dr. James Naughton and Miss E. I. Thompson. I am indebted to Mr. Clifford Shepley, artist to the University of Edinburgh, for his most artistic illustrations drawn from many photographs. Miss E. Cruickshank gave much secretarial assistance.

The survey would never have been made without the stimulation and guidance of Professor R. W. B. Ellis. I am most grateful to him, also, for his help in the preparation of this paper.

\section{REFERENCES}

Asher, P. and Schonell, F. E. (1950). Archives of Disease in Childhood, 25, 360 .

Bobath, K. and Bobath, B. (1950). Brit. J. phys. Med., 13, 121. Ingram, T. T. S. (1955). Archives of Disease in Childhood, 30, 85. Little, W. J. (1862). Trans. obstet. Soc. Lond., 3, 293. Lovett, R. W. (1888). Boston med. Surg. J., 118, 641. Oster, W. (1889). The Cerebral Palsies of Children. London. Parrot, J. (1873). Arch. Physiol. norm. path., 5, 59. 